Sādhanā Vol. 38, Part 3, June 2013, pp. 491-511. (C) Indian Academy of Sciences

\title{
Predictive modelling of noise level generated during sawing of rocks by circular diamond sawblades
}

\author{
IZZET KARAKURT*, GOKHAN AYDIN and KERIM AYDINER \\ Department of Mining Engineering, Karadeniz Technical University, Trabzon 61080, \\ Turkey \\ e-mail: karakurt@ktu.edu.tr
}

MS received 24 May 2012; revised 3 September 2012; accepted 15 September 2012

\begin{abstract}
This paper presents an experimental and statistical study on noise level generated during of rock sawing by circular diamond sawblades. Influence of the operating variables and rock properties on the noise level are investigated and analysed. Statistical analyses are then employed and models are built for the prediction of noise levels depending on the operating variables and the rock properties. The derived models are validated through some statistical tests. It is found that increasing of peripheral speed, traverse speed and cutting depth result in an increase in noise levels. On the other hand, a decreasing trend for noise levels is initially observed with the increasing of flow rate of cooling fluid. It is also determined that there are moderate correlations between uniaxial compressive strength, density and noise levels. Furthermore, the modelling results reveal that the predictive models have high potentials as guidance for practical applications.
\end{abstract}

Keywords. Diamond sawblades; granite; noise level; predictive model.

\section{Introduction}

Various rock cutting works are carried out in mining as well as civil engineering applications by means of different machines and cutting tools. Circular diamond sawblades are one of the most used cutting tools that have been widely used in sawing of natural stones throughout the world (Atıcı \& Ersoy 2009; Rojek et al 2011). Sawing performance and life of a circular sawblade are affected by many factors including diamond and matrix properties, operating variables, sawing modes (up-cutting, down-cutting), physico-mechanical and mineralogical properties of the rock to be cut, condition of the sawing machine and the skills of the operator (Fener et al 2007; Buyuksagis 2007). Basically, the parameters affecting the performance and life of the sawblades indicate the complexity of these tools. In spite of the complexity of the system, a considerable amount of work has been conducted and published by many researchers in the relevant literature to understand the cutting tool itself and mechanism behind the phenomena. In recent years, due to the importance in cost estimation and planning of the plants, the prediction of the cutting

${ }^{*}$ For correspondence 
performance of circular diamond sawblades in sawing of natural stones has been the subject of considerable research interest. Kahraman et al (2004), Ersoy \& Atıc1 (2004), Buyuksagis (2007), Y1lmaz et al (2011), Yurdakul \& Akdaş (2012), and Ataei et al (2012) are among the known attempts studying the prediction of cutting performance of circular diamond sawblades in sawing of rocks.

Noise is an effect arising in the rock cutting process as well as in other industries where machining and/or processing of materials is conducted. High noise levels not only affect the workers' life, but also contribute rather serious problems to the working environment. Additionally, noise level may be a good indicator for tool performance against cutting. Therefore, alternative designs aiming at minimizing the noise generated during the sawing of rocks have recently gained attention. Furthermore, from the relevant literature, it can be clearly concluded that although there are many studies focusing on the investigation of the sawing performance of a circular sawblade including the prediction studies, almost none of them has investigated the correlations between sawability and noise level generated during the sawing of rocks. Therefore, the lack of investigation on the sawing performance of a circular sawblade in terms of the noise level has led to conduct the current study. It may be important to note that it is very hard to build predictive models for the performance indicators including noise levels, valid for all rock types since the effects of operating variables and materials properties on performance indicators vary from one rock type to another. Hence, in the current study, a group of rock (granitic rock) was sawn using a circular diamond sawblades. Effects of the operating and material properties on the noise level were investigated through correlation analysis. Predictive models were then developed by regression analysis to estimate the noise level from both operating variables and material properties separately. Finally, the derived models were validated through some statistical tests.

\section{Experimental study}

\subsection{Material characterization}

Nine granitic rocks having different percentages of minerals and grain size distributions, and merchandised both in Turkey and other parts of the World, were selected from a stone processing plant. The selected rocks include Verde Butterfly (R1), Giallo Fiorito (R2), Porto Rosa (R3), Crema Lal (R4), Giresun Vizon (R5), Balaban Green (R6), Bergama Gri (R7), Nero Zimbabwe (R8) and Star Galaxy (R9). The granite samples have a length of $30 \times 10 \times 3 \mathrm{~cm}$ section.

Some physico-mechanical properties of the selected granites are given in table 1 . It may be important to note that in practice, there are serious difficulties of preparing and testing hard rocks including granites for their mechanical properties such as uniaxial compressive and bending strength. For these reasons, the uniaxial compressive and flexural strengths of the tested rocks were provided by the stone processing company where the selected rocks were provided. Density $\left(\mathrm{kN} / \mathrm{m}^{3}\right)$, water absorption by volume $(\%)$, porosity $(\%)$, ultrasonic velocity $(\mathrm{m} / \mathrm{s})$, Shore hardness, Schmidt hammer hardness, Cerchar abrasion index (HV), microhardness and Mohs hardness were determined according to related ISRM (1981) suggested methods.

Thin section studies were prepared for each rock sample and examined using the polarizing microscope to determine the petrographic composition and grain-size of the minerals of tested rocks (see figure 1). Additionally, polished hand specimens were examined for grain size characterization. This was carried out for especially coarse-grained rock samples. Petrographic descriptions, mineralogical compositions and grain size ranges of the tested rocks are given in 
Table 1. Mechanical and intact properties of rocks used in the sawing tests.

\begin{tabular}{|c|c|c|c|c|c|c|c|c|c|}
\hline Rock properties & $\mathrm{R} 1$ & $\mathrm{R} 2$ & R3 & $\mathrm{R} 4$ & R5 & R6 & R7 & $\mathrm{R} 8$ & R9 \\
\hline $\begin{array}{l}\text { Uniaxial strength } \\
\text { (MPa) }\end{array}$ & 191.18 & 167.65 & 107.94 & 231.34 & 132.35 & 145.00 & 92.65 & 242.6 & 201.47 \\
\hline Density $\left(\mathrm{kN} / \mathrm{m}^{3}\right)$ & 27.60 & 26.60 & 26.40 & 25.9 & 26.7 & 26.6 & 26.09 & 30.30 & 28.40 \\
\hline $\begin{array}{l}\text { Bending strength } \\
\text { (MPa) }\end{array}$ & 13.14 & 22.06 & 15.00 & 19.42 & 18.14 & 15.20 & 14.90 & 24.02 & 19.61 \\
\hline $\begin{array}{c}\text { Water absorption } \\
\text { by volume }(\%)\end{array}$ & 0.20 & 0.28 & 0.30 & 0.86 & 0.20 & 0.19 & 0.30 & 0.14 & 0.10 \\
\hline Porosity $(\%)$ & 1.50 & 0.80 & 1.50 & 1.50 & 3.30 & 2.20 & 1.80 & 0.30 & 1.00 \\
\hline $\begin{array}{l}\text { Ultrasonic } \\
\text { Velocity }(\mathrm{m} / \mathrm{s})\end{array}$ & 4130 & 3917 & 4196 & 4140 & 5866 & 4849 & 4836 & 6054 & 6863 \\
\hline $\begin{array}{l}\text { Cerchar abrasion } \\
\text { Index }\end{array}$ & 4.348 & 4.166 & 4.508 & 5.2 & 3.868 & 4.356 & 4.622 & 3.412 & 4.29 \\
\hline $\begin{array}{l}\text { Schmidt hammer } \\
\text { hardness }\end{array}$ & 47 & 48 & 51 & 56 & 54 & 55 & 54 & 64 & 65 \\
\hline $\begin{array}{l}\text { Microhardness } \\
(\mathrm{HV})\end{array}$ & 502.04 & 543.47 & 538.73 & 539.55 & 505.5 & 559.03 & 537.93 & 501.84 & 463.18 \\
\hline Shore hardness & 72.65 & 73.55 & 81.85 & 75.6 & 83.1 & 75.15 & 71.35 & 71.9 & 60.8 \\
\hline Mohs hardness & 6.1 & 5.7 & 6.0 & 4.5 & 6.0 & 6.0 & 6.3 & 6.2 & 5.8 \\
\hline
\end{tabular}

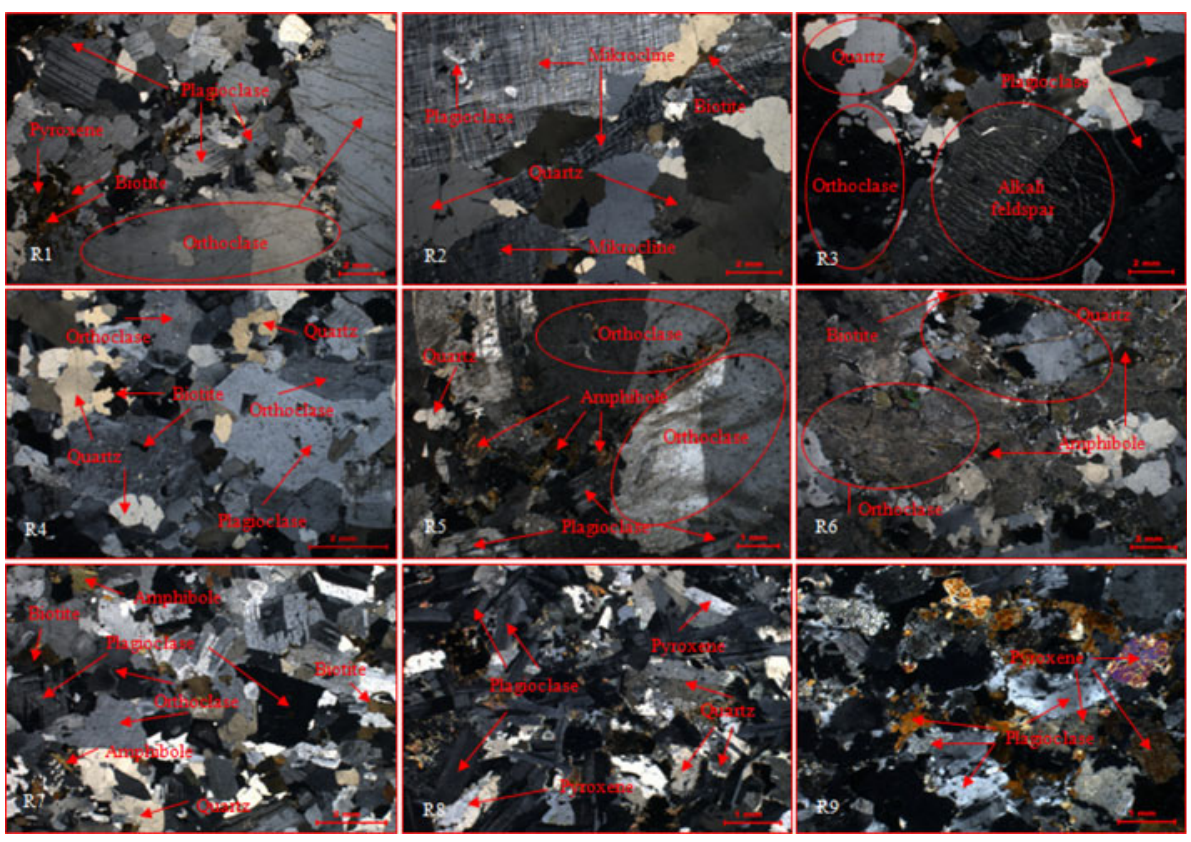

Figure 1. Photomicrographs of the rocks tested. 


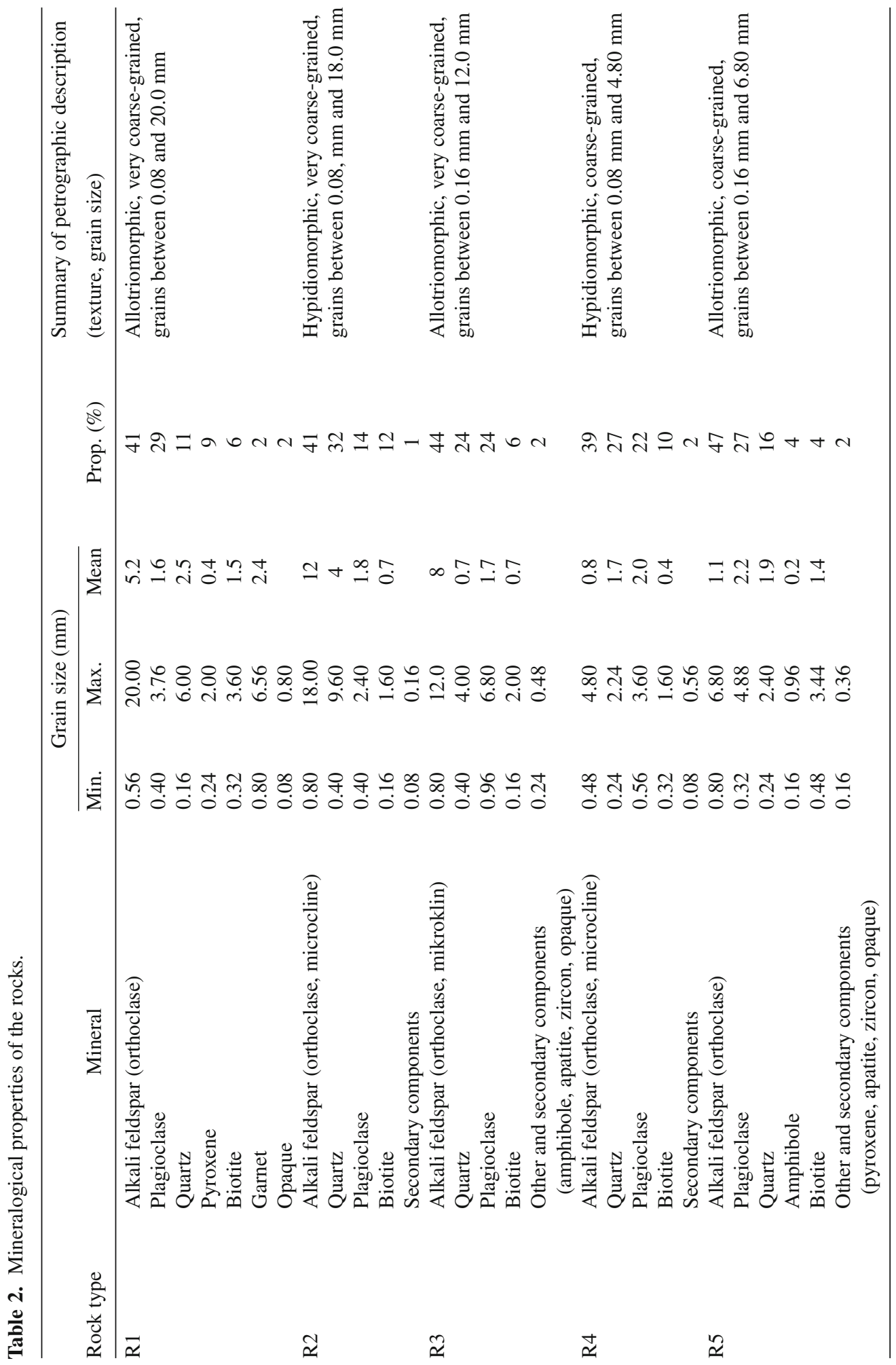




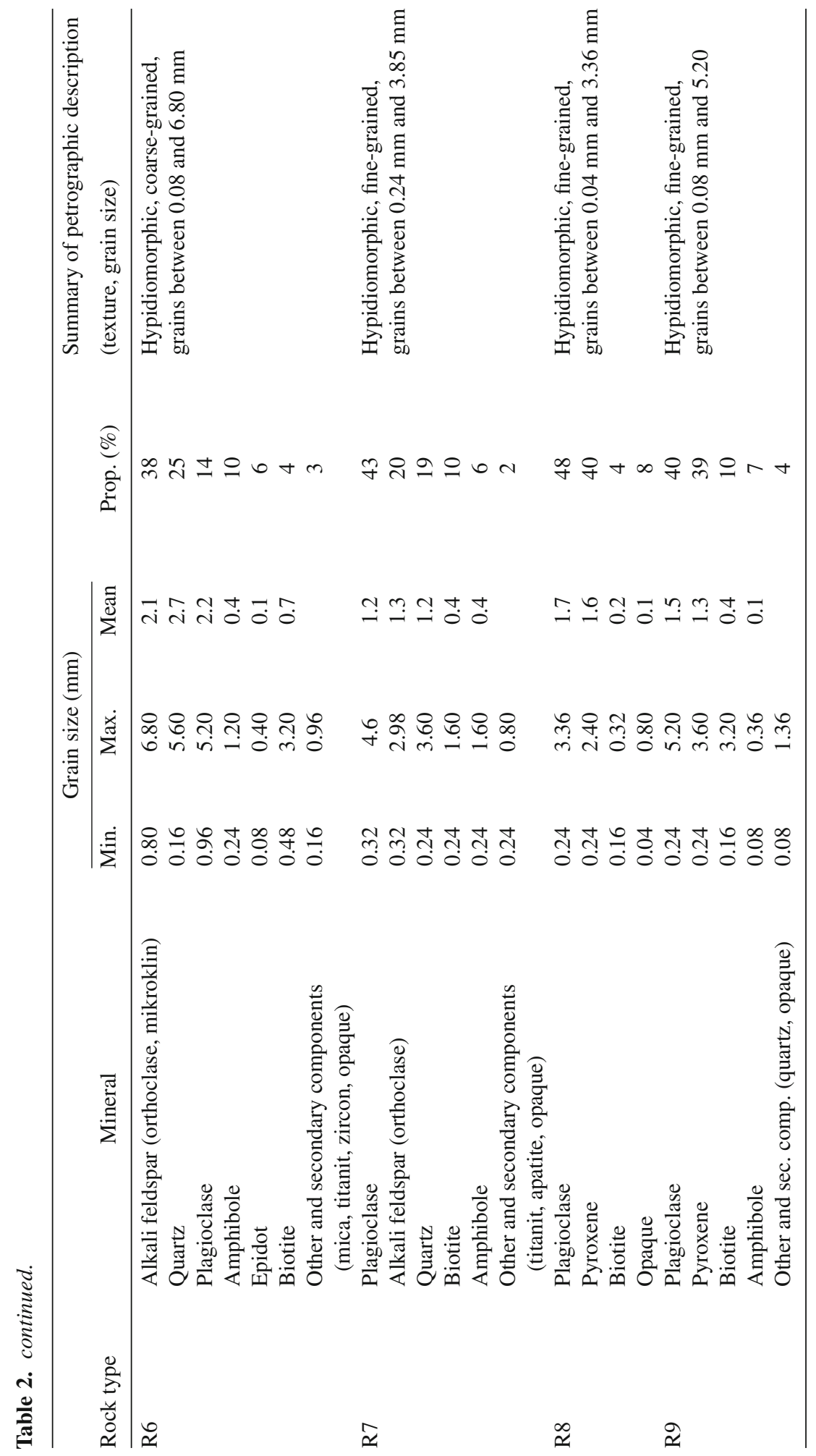




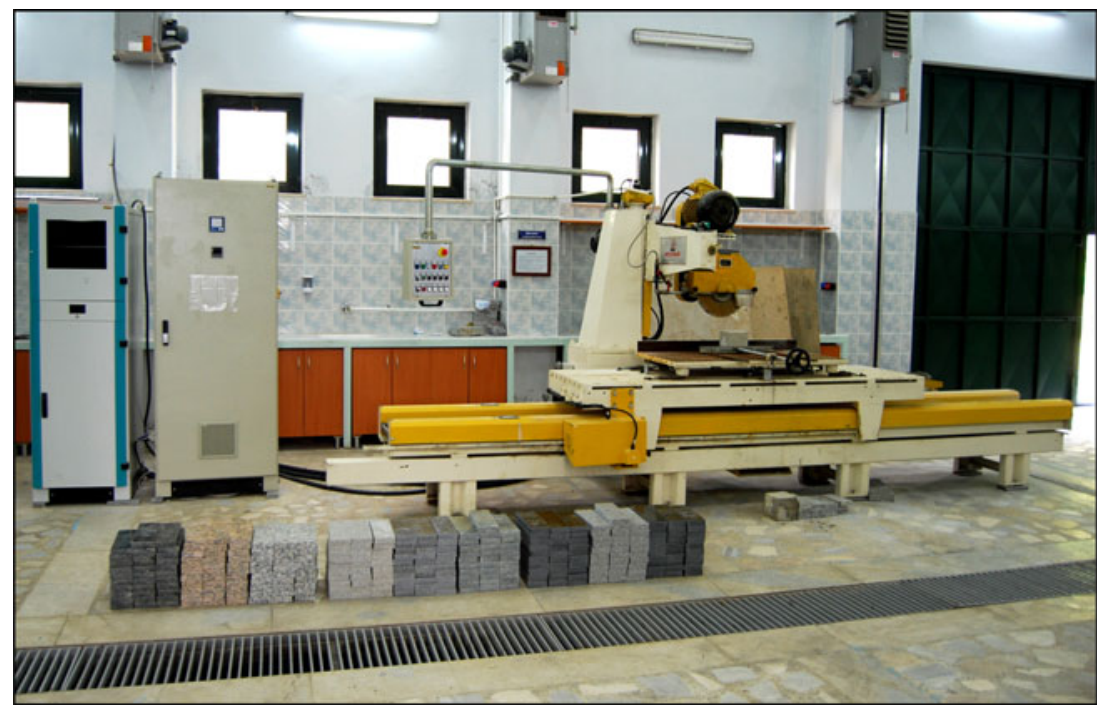

Figure 2. Experimental set-up.

table 2. As can be followed from table 2; quartz, K-feldspar, plagioclase and biotite were the main rock-forming minerals in all samples, varying in their percentage contents.

\subsection{Experimental procedure}

Sawing experiments were conducted on a fully-instrumented cutting machine (see figure 2). The cutting machine consists mainly of three major sub-systems; a cutting unit, instrumentation and a personal computer (PC). The diamond sawblade used in the tests was of $40 \mathrm{~cm}$ diameter, having 28 impregnated diamond segments (circumferential length $40 \mathrm{~mm}$, width $3.5 \mathrm{~mm}$ and height $10 \mathrm{~mm}$ ). The diamonds were sized at 40/50 US mesh with a concentration of 30 which is recommended for the sawing of hard materials. Disc movements forward-backward in the horizontal plane and up-down in the vertical plane were driven with two $0.75 \mathrm{~kW}$ ac motors, while the turn of the disc were driven with $4 \mathrm{~kW}$ ac motor. Moreover, $0.75 \mathrm{~kW}$ ac motor was used to move the wagon in the cutting line. Vertical, horizontal, axial forces were measured using sensors, load cells, transducers and an encoder in the monitoring system. All movements of the cutting machine were controlled by a computer and industrial electronic cards. Transmissions to the computer were carried out using processing software.

Table 3. Operating variables and their levels.

\begin{tabular}{|c|c|c|c|c|c|}
\hline \multirow{2}{*}{$\frac{\text { Operating variables }}{\text { Peripheral speed }(\mathrm{m} / \mathrm{s})}$} & \multicolumn{5}{|c|}{ Level } \\
\hline & 25 & 30 & 35 & 40 & 45 \\
\hline Traverse speed $(\mathrm{cm} / \mathrm{min})$ & 40 & 50 & 60 & 70 & 80 \\
\hline Cutting depth $(\mathrm{cm})$ & 0.5 & 1.0 & 1.5 & 2.0 & 2.5 \\
\hline Flow rate of cooling fluid $(\mathrm{ml} / \mathrm{s})$ & 50 & 100 & 150 & 200 & 250 \\
\hline
\end{tabular}


Table 4. Experimental layout.

\begin{tabular}{lcccc}
\hline $\begin{array}{l}\text { Number of } \\
\text { experiment }\end{array}$ & $\begin{array}{c}\text { Peripheral speed } \\
(\mathrm{m} / \mathrm{s})\end{array}$ & $\begin{array}{c}\text { Traverse speed } \\
(\mathrm{cm} / \mathrm{min})\end{array}$ & $\begin{array}{c}\text { Cutting depth } \\
(\mathrm{cm})\end{array}$ & $\begin{array}{c}\text { Flow rate of cooling fluid } \\
(\mathrm{ml} / \mathrm{s})\end{array}$ \\
\hline 1 & $\mathbf{2 5}$ & 60 & 2.0 & 150 \\
2 & $\mathbf{3 0}$ & 60 & 2.0 & 150 \\
3 & $\mathbf{3 5}$ & 60 & 2.0 & 150 \\
4 & $\mathbf{4 0}$ & 60 & 2.0 & 150 \\
5 & $\mathbf{4 5}$ & 60 & 2.0 & 150 \\
6 & 35 & $\mathbf{4 0}$ & 2.0 & 150 \\
7 & 35 & $\mathbf{5 0}$ & 2.0 & 150 \\
8 & 35 & $\mathbf{6 0}$ & 2.0 & 150 \\
9 & 35 & $\mathbf{7 0}$ & 2.0 & 150 \\
10 & 35 & $\mathbf{8 0}$ & 2.0 & 150 \\
11 & 35 & 60 & $\mathbf{0 . 5}$ & 150 \\
12 & 35 & 60 & $\mathbf{1 . 0}$ & 150 \\
13 & 35 & 60 & $\mathbf{1 . 5}$ & 150 \\
14 & 35 & 60 & $\mathbf{2 . 0}$ & 150 \\
15 & 35 & 60 & $\mathbf{2 . 5}$ & 150 \\
16 & 35 & 60 & 2.0 & $\mathbf{5 0}$ \\
17 & 35 & 60 & 2.0 & $\mathbf{1 0 0}$ \\
18 & 35 & 60 & 2.0 & $\mathbf{1 5 0}$ \\
19 & 35 & 60 & 2.0 & $\mathbf{2 0 0}$ \\
20 & 35 & 60 & 2.0 & $\mathbf{2 5 0}$ \\
\hline & & & & \\
\hline
\end{tabular}

In order to determine the levels of operating variables for the study, some preliminary cutting tests were conducted by considering instructions of diamond disc manufacturers and the relevant studies in the literature. Consequently, valid for all types of tested rocks, operating variables

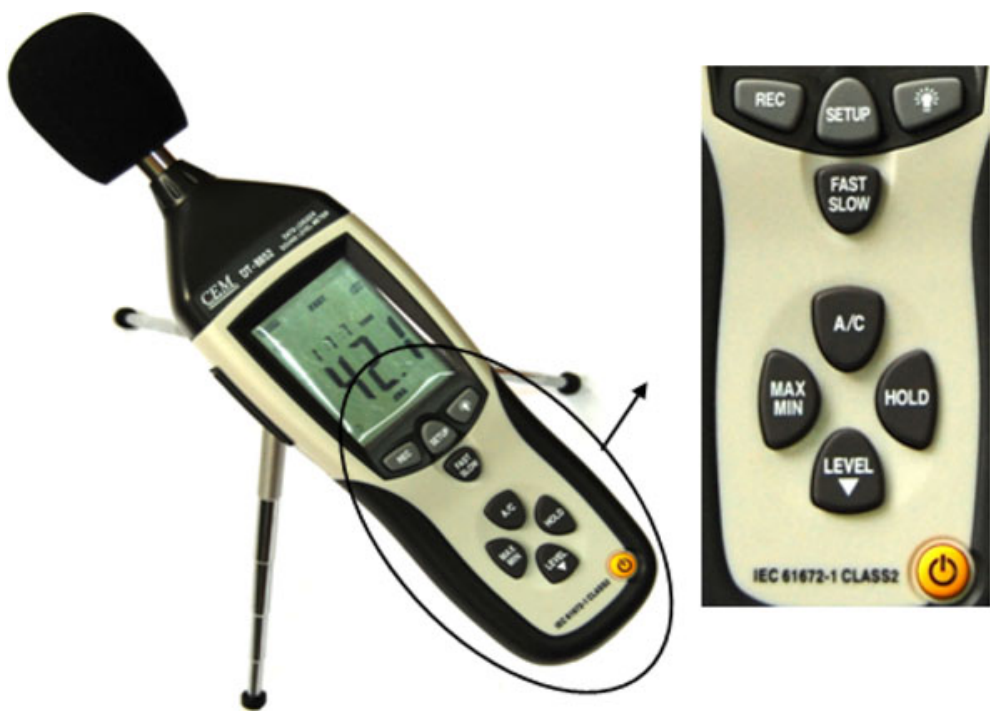

Figure 3. Noise level meter used in the study. 
Table 5. Specifications of the noise level meter used in the study.

\begin{tabular}{ll}
\hline Standard applied & \multicolumn{1}{c}{ IEC61672-1 Type 2, ANSI S1.4 Type2 } \\
\hline Accuracy & $\pm 1.4 \mathrm{~dB}$ \\
Frequency range & $31.5 \mathrm{HZ} \sim 8 \mathrm{KHZ}$ \\
Dynamic range & $50 \mathrm{~dB}$ \\
Level ranges & $\mathrm{LO}: 30 \mathrm{~dB} \sim 80 \mathrm{~dB}$ Med:50dB $\sim 100 \mathrm{~dB}$ \\
& Hi:80dB $\sim 130 \mathrm{~dB}$ Auto:30dB $\sim 130 \mathrm{~dB}$ \\
Time weighting & FAST $(125 \mathrm{mS})$, SLOW $(1 \mathrm{~s})$ \\
Microphone & $1 / 2$ inch electrets condenser microphone \\
Resolution & $0.1 \mathrm{~dB}$ \\
Display update & 2 times $/ \mathrm{sec}$ \\
Analog output & AC/DC outputs, AC $=1 \mathrm{Vrms,} \mathrm{DC}=10 \mathrm{mV} / \mathrm{dB}$ \\
\hline
\end{tabular}

were selected as given in table 3 and all operating variables was varied at the levels presented in table 4. Rock samples were sawn through their lengths and all sawing experiments were performed by the same cutting mode (down-cutting mode).

A noise level meter modelled as 'DT-8852' was used to measure the noise level during the cutting process (see figure 3 ). The main specifications of the noise level meter are given in table 5 . The equipment has a screen showing the noise level values and the max, min and instantaneous noise levels during the cutting can be seen on this screen. The data obtained was transported to a PC and the average noise level for every cutting was reported through a software interface as shown in figure 4.

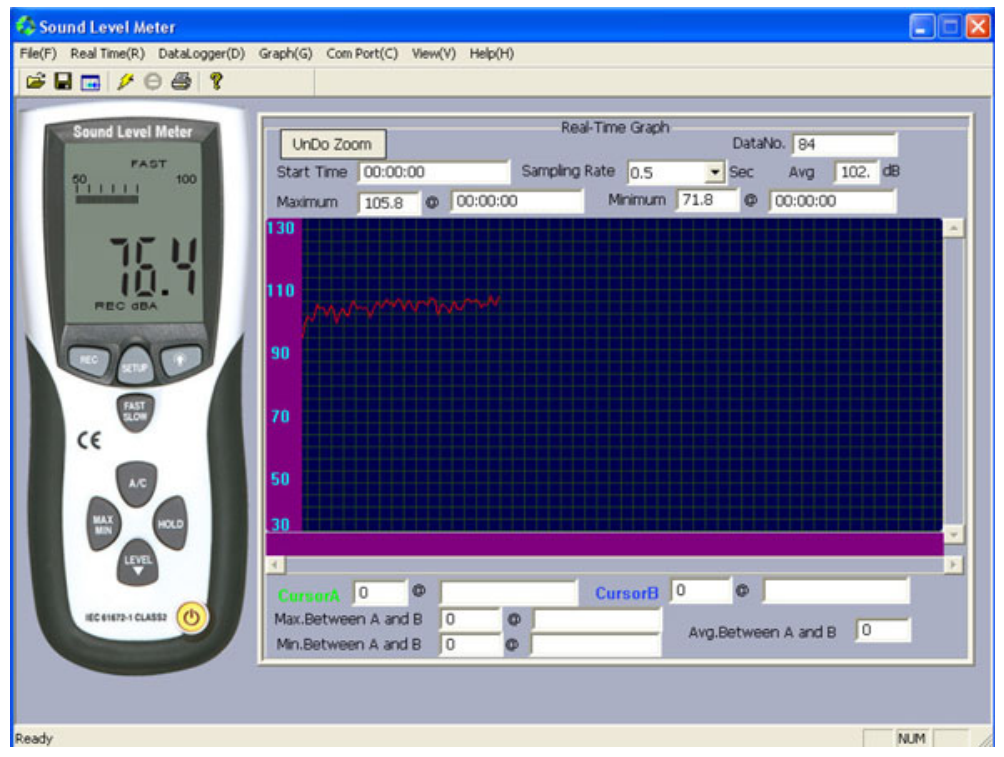

Figure 4. Software interface of the noise level meter. 


\section{Results and discussion}

To evaluate the experimental results, firstly, the influence of the operating variables on noise level was analysed and contribution of each operating variable on the noise level was determined. Afterwards, to determine the best empirical correlations between material properties and noise level, bivariate correlation technique was then applied. Further, models were developed using multiple regression analysis to predict the noise level from both operating variables and material properties. Regression analysis was performed for the experimental data obtained from $19^{\text {th }}$ test to determine the most important material properties in terms of the noise level. In other words, a constant specific removal rate of $120 \mathrm{~cm}^{2} / \mathrm{min}$ was employed throughout the experiments so that all granite types could be easily sawn within the available power limits of the circular diamond sawblades. The same cutting rate can enable a direct comparison of results obtained for all the rock samples. All statistical analysis was carried out by a statistical software called 'SPSS' (Statistical Package for the Social Sciences). Additionally, the models derived from the regression analysis were validated through some statistical tests.

\subsection{Influence of the operating variables}

Influences of the operating variables on the noise level were depicted in figure 5. It can be stated that increasing of peripheral speed, traverse speed and cutting depth resulted in an increase in noise levels recorded for all rocks. Unlike the behaviour of other operating variables, a decreasing trend for noise levels reported for all rocks was initially observed with the increasing of flow rate of cooling fluid. Further increase in flow rate of cooling fluid resulted in steady changes in noise levels. Moreover, it can be stated that the noise levels increased as a result of the increase in specific removal rate that is the quantity of the material sawn in unit time or the area cut per unit time as shown in figure 6.
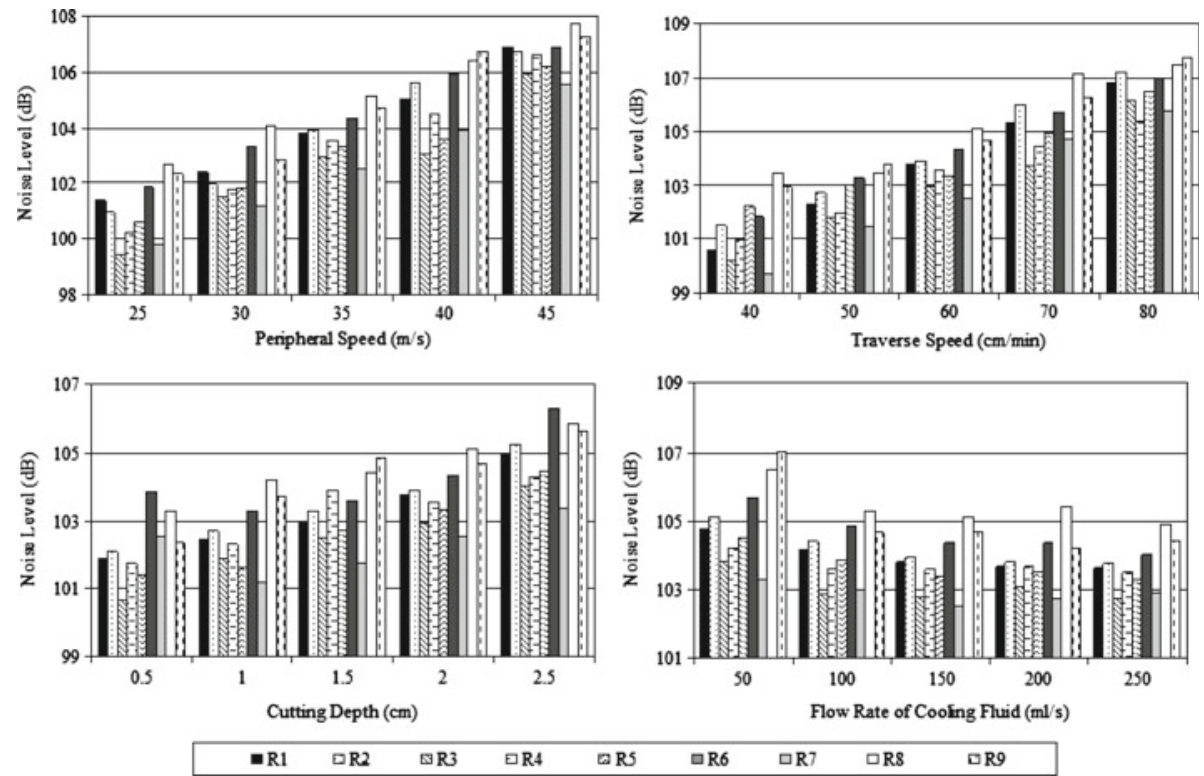

Figure 5. Relation between operating variables and noise level. 


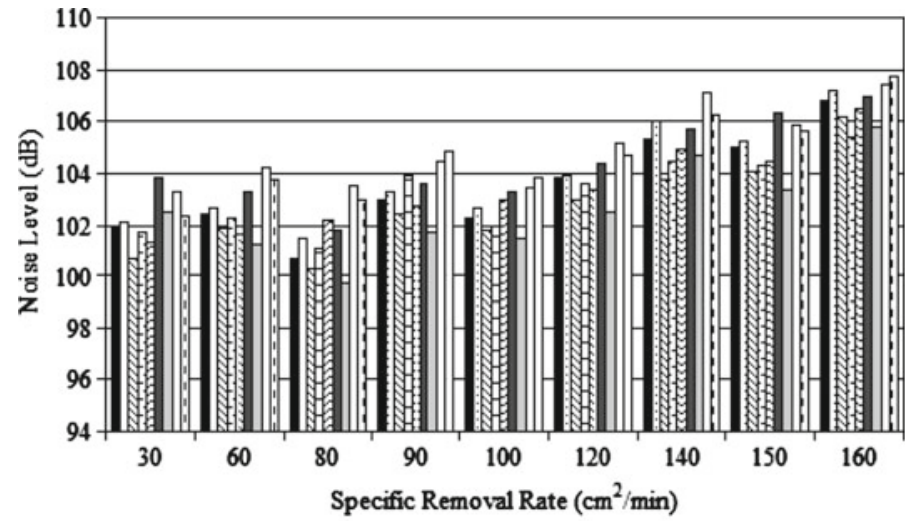

$\begin{array}{lllllllll}\mathbf{R} 1 & \square R 2 & \mathbb{R} 3 & \square \mathrm{R} 4 & \mathbf{R} 5 & \square \mathrm{R} 6 & \square \mathrm{R} 7 & \square \mathrm{R} 8 & \square \mathrm{R} 9\end{array}$

Figure 6. Relation between specific removal rate and noise level.
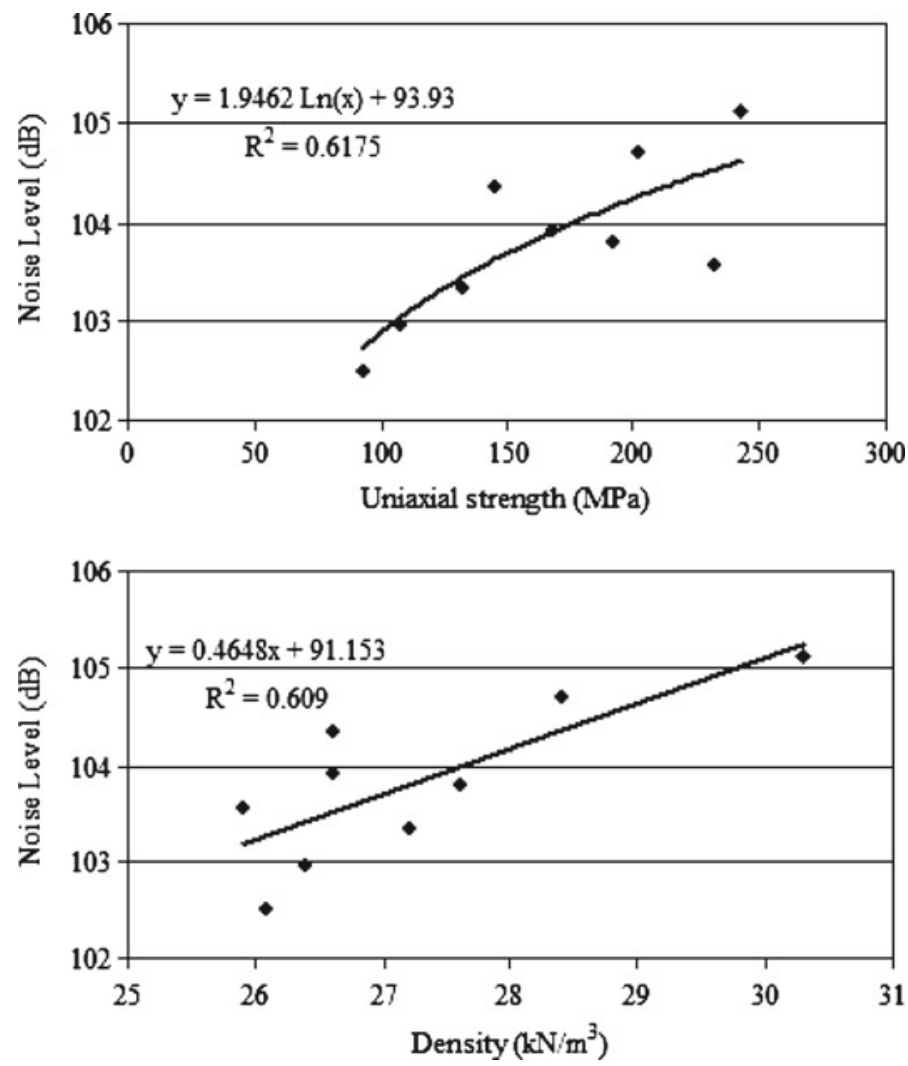

Figure 7. Main rock properties affecting the noise level. 


\subsection{Influence of the material properties}

The relationships between physico-mechanical and mineralogical properties and noise levels were investigated on the basis of the statistical approaches such as linear, logarithmic, exponent and exponential, and the best relations established were depicted in figure 7 and given in tables 6 and 7 , respectively. It can be concluded from the figure and tables that there are moderate correlations between uniaxial compressive strength, density and noise level. In other words, it can be said that the rocks having higher uniaxial compressive strengths and densities may generate higher noises during cutting process.

\subsection{Predictive models for noise level}

To build models for prediction of noise level from operating variables, multiple regression analysis was performed using the dependent (noise level) and independent variables (operating variables). Regression analysis is a statistical methodology for predicting values of one or more dependent variables from a collection of (independent) variable values (Muro \& Tran 2004).

The dependent and independent variables are denoted as following; NL: noise level, A: peripheral speed, B: workpiece traverse speed, C: cutting depth, D: flow rate of cooling fluid. The models developed for the estimation of noise level from operating variables for each rock are presented below together with the contribution rates (CRs) of each operating variable involved in the models to noise level (equations from 1 to 9). From analysis of contribution rates (CR), it was concluded that the peripheral speed was determined as the most significant operating variables affecting the noise level. The peripheral speed was followed by the traverse speed, cutting depth and flow rate of cooling fluid (for some rocks), respectively. It can be also concluded that among the operating variables, the flow rate of cooling fluid was the operating variables that has less effect on the noise level.

$$
\begin{aligned}
& \mathbf{N L}_{\mathbf{R} 1}(\mathbf{d B})=82.9560+0.2734 \mathrm{~A}+0.1545 \mathrm{~B}+1.4859 \mathrm{C}-0.0056 \mathrm{D} \\
& \mathbf{C R}(\boldsymbol{\%}): \mathrm{A}: 33.69, \mathrm{~B}: 38.07, \mathrm{C}: 21.29, \mathrm{D}: 6.95 .
\end{aligned}
$$

$$
\begin{aligned}
& \mathbf{N L}_{\mathbf{R} 2}(\mathbf{d B})=82.8019+0.3040 \mathrm{~A}+0.1461 \mathrm{~B}+1.4743 \mathrm{C}-0.0067 \mathrm{D} \\
& \mathbf{C R}(\boldsymbol{\%}): \mathrm{A}: 36.44, \mathrm{~B}: 35.02, \mathrm{C}: 20.55, \mathrm{D}: 7.98
\end{aligned}
$$

$$
\begin{aligned}
& \mathbf{N L}_{\mathbf{R} 3}(\mathbf{d B})=81.5591+0.2922 \mathrm{~A}+0.1389 \mathrm{~B}+1.4037 \mathrm{C} \\
& \mathbf{C R}(\boldsymbol{\%}): \mathrm{A}: 39.85, \mathrm{~B}: 37.88, \mathrm{C}: 22.27
\end{aligned}
$$

$$
\begin{aligned}
& \mathbf{N L}_{\mathbf{R} 4}(\mathbf{d B})=83.5386+0.3136 \mathrm{~A}+0.1138 \mathrm{~B}+1.0748 \mathrm{C} \\
& \mathbf{C R}(\boldsymbol{\%}): \mathrm{A}: 47.07, \mathrm{~B}: 34.16, \mathrm{C}: 18.76 .
\end{aligned}
$$

$$
\begin{aligned}
& \mathbf{N L}_{\mathbf{R} 5}(\mathbf{d B})=84.8860+0.2600 \mathrm{~A}+0.1049 \mathrm{~B}+1.6721 \mathrm{C} \\
& \mathbf{C R}(\%): \mathrm{A}: 39.14, \mathrm{~B}: 31.58, \mathrm{C}: 29.28
\end{aligned}
$$


Table 6. Regression analysis concerning the physico-mechanical properties and noise level.

\begin{tabular}{|c|c|c|}
\hline Physico-mechanical properties & Regression equation & $\mathrm{R}^{2}$ \\
\hline \multirow[t]{4}{*}{ Uniaxial strength (MPa) } & $\mathrm{NL}=0.0119 \mathrm{x}+101.81$ & 0.5730 \\
\hline & $\mathrm{NL}=1.9462 \operatorname{Ln}(\mathrm{x})+93.93$ & 0.6175 \\
\hline & $\mathrm{NL}=94.374 \mathrm{x}^{0.0188}$ & 0.6189 \\
\hline & $\mathrm{NL}=101.83 \mathrm{e}^{0.0001 \mathrm{x}}$ & 0.5738 \\
\hline \multirow[t]{4}{*}{ Density $\left(\mathrm{kN} / \mathrm{m}^{3}\right)$} & $\mathrm{NL}=0.4648 \mathrm{x}+91.153$ & 0.6090 \\
\hline & $\mathrm{NL}=13.032 \operatorname{Ln}(\mathrm{x})+60.76$ & 0.6107 \\
\hline & $\mathrm{NL}=68.632 \mathrm{x}^{0.1253}$ & 0.6082 \\
\hline & $\mathrm{NL}=91.918 \mathrm{e}^{0.0045 \mathrm{x}}$ & 0.6064 \\
\hline \multirow[t]{4}{*}{ Bending strength (MPa) } & $\mathrm{NL}=0.1347 \mathrm{x}+101.39$ & 0.3548 \\
\hline & $\mathrm{NL}=2.3207 \operatorname{Ln}(\mathrm{x})+97.152$ & 0.3238 \\
\hline & $\mathrm{NL}=97.366 \mathrm{x}^{0.0223}$ & 0.3231 \\
\hline & $\mathrm{NL}=101.42 \mathrm{e}^{0.0013 \mathrm{x}}$ & 0.3549 \\
\hline \multirow[t]{4}{*}{ Water absorption by volume (\%) } & $\mathrm{NL}=-1.3128 \mathrm{x}+104.18$ & 0.1286 \\
\hline & $\mathrm{NL}=-0.7853 \operatorname{Ln}(\mathrm{x})+102.68$ & 0.3288 \\
\hline & $\mathrm{NL}=102.68 \mathrm{x}^{-0.0075}$ & 0.3275 \\
\hline & $\mathrm{NL}=104.18 \mathrm{e}^{-0.0126 \mathrm{x}}$ & 0.1278 \\
\hline \multirow[t]{4}{*}{ Porosity (\%) } & $\mathrm{NL}=-0.4908 \mathrm{x}+104.57$ & 0.2624 \\
\hline & $\mathrm{NL}=-0.7803 \operatorname{Ln}(\mathrm{x})+104.01$ & 0.4176 \\
\hline & $\mathrm{NL}=104.01 \mathrm{x}^{-0.0075}$ & 0.4154 \\
\hline & $\mathrm{NL}=104.57 \mathrm{e}^{-0.0047 x}$ & 0.2611 \\
\hline \multirow[t]{4}{*}{ Schmidt hammer hardness } & $\mathrm{NL}=0.0801 \mathrm{x}+99.412$ & 0.3658 \\
\hline & $\mathrm{NL}=4.2743 \operatorname{Ln}(\mathrm{x})+86.714$ & 0.3336 \\
\hline & $\mathrm{NL}=88.102 \mathrm{x}^{0.041}$ & 0.3312 \\
\hline & $\mathrm{NL}=99.517 \mathrm{e}^{0.0008 \mathrm{x}}$ & 0.3632 \\
\hline \multirow[t]{4}{*}{ Ultrasonic Velocity $(\mathrm{m} / \mathrm{s})$} & $\mathrm{NL}=0.0004 \mathrm{x}+101.76$ & 0.2671 \\
\hline & $\mathrm{NL}=2.0426 \operatorname{Ln}(\mathrm{x})+86.457$ & 0.2452 \\
\hline & $\mathrm{NL}=87.884 \mathrm{x}^{0.0196}$ & 0.2434 \\
\hline & $\mathrm{NL}=101.79$ e4E-06x & 0.2653 \\
\hline \multirow[t]{4}{*}{ Shore hardness } & $\mathrm{NL}=-0.0647 \mathrm{x}+108.6$ & 0.2565 \\
\hline & $\mathrm{NL}=-4.6242 \operatorname{Ln}(\mathrm{x})+123.7$ & 0.2544 \\
\hline & $\mathrm{NL}=125.67 \mathrm{x}^{-0.0444}$ & 0.2533 \\
\hline & $\mathrm{NL}=108.7 \mathrm{e}^{-0.0006 \mathrm{x}}$ & 0.2554 \\
\hline \multirow[t]{4}{*}{ Cerchar abrasion Index } & $\mathrm{NL}=-0.9075 \mathrm{x}+107.72$ & 0.2932 \\
\hline & $\mathrm{NL}=-3.9655 \operatorname{Ln}(\mathrm{x})+109.58$ & 0.3133 \\
\hline & $\mathrm{NL}=109.72 \mathrm{x}^{-0.0381}$ & 0.3118 \\
\hline & $\mathrm{NL}=107.78 \mathrm{e}^{-0.0087 \mathrm{x}}$ & 0.2918 \\
\hline \multirow[t]{4}{*}{ Microhardness (HV) } & $\mathrm{NL}=-0.0122 \mathrm{x}+110.18$ & 0.1957 \\
\hline & $\mathrm{NL}=-6.3592 \operatorname{Ln}(\mathrm{x})+143.59$ & 0.2035 \\
\hline & $\mathrm{NL}=152.18 \mathrm{x}^{-0.0612}$ & 0.2029 \\
\hline & $\mathrm{NL}=110.36 \mathrm{e}^{-0.0001 \mathrm{x}}$ & 0.1952 \\
\hline \multirow[t]{4}{*}{ Mohs hardness } & $\mathrm{NL}=0.0086 \mathrm{x}+103.76$ & $3 \mathrm{E}-05$ \\
\hline & $\mathrm{NL}=0.1617 \operatorname{Ln}(\mathrm{x})+103.53$ & 0.0004 \\
\hline & $\mathrm{NL}=103.55 \mathrm{x}^{0.0014}$ & 0.0003 \\
\hline & $\mathrm{NL}=103.77 \mathrm{e}^{5 \mathrm{E}-05 \mathrm{x}}$ & $1 \mathrm{E}-05$ \\
\hline
\end{tabular}


Table 7. Regression analysis concerning the mineralogical properties and noise level.

\begin{tabular}{|c|c|c|}
\hline Mineralogical properties & Regression equation & $\mathrm{R}^{2}$ \\
\hline \multirow[t]{4}{*}{ Plagioclase content (\%) } & $\mathrm{NL}=0.0117 \mathrm{x}+103.47$ & 0.0298 \\
\hline & $\mathrm{NL}=0.1461 \operatorname{Ln}(\mathrm{x})+103.33$ & 0.0063 \\
\hline & $\mathrm{NL}=103.35 \mathrm{x}^{0.0014}$ & 0.0058 \\
\hline & $\mathrm{NL}=103.48 \mathrm{e}^{0.0001 \mathrm{x}}$ & 0.0287 \\
\hline \multirow[t]{2}{*}{ Feldspar content $(\%)$} & $\mathrm{NL}=-0.0252 \mathrm{x}+104.57$ & 0.3210 \\
\hline & $\mathrm{NL}=104.56 \mathrm{e}^{-0.0002 \mathrm{x}}$ & 0.3184 \\
\hline \multirow[t]{2}{*}{ Quarts content (\%) } & $\mathrm{NL}=-0.0399 \mathrm{x}+104.49$ & 0.3062 \\
\hline & $\mathrm{NL}=104.49 \mathrm{e}^{-0.0004 \mathrm{x}}$ & 0.3041 \\
\hline \multirow[t]{4}{*}{ Biotite content (\%) } & $\mathrm{NL}=-0.0595 \mathrm{x}+104.25$ & 0.0516 \\
\hline & $\mathrm{NL}=-0.5022 \operatorname{Ln}(\mathrm{x})+104.77$ & 0.0755 \\
\hline & $\mathrm{NL}=104.77 \mathrm{x}^{-0.0048}$ & 0.0753 \\
\hline & $\mathrm{NL}=104.24 \mathrm{e}^{-0.0006 \mathrm{x}}$ & 0.0514 \\
\hline \multirow[t]{4}{*}{ Mean grain size of plagioclase (mm) } & $\mathrm{NL}=0.5186 \mathrm{x}+102.89$ & 0.0421 \\
\hline & $\mathrm{NL}=1.0906 \operatorname{Ln}(\mathrm{x})+103.21$ & 0.0647 \\
\hline & $\mathrm{NL}=103.2 \mathrm{x}^{0.0106}$ & 0.0660 \\
\hline & $\mathrm{NL}=102.88 \mathrm{e}^{0.0051 \mathrm{x}}$ & 0.0431 \\
\hline \multirow[t]{2}{*}{ Mean grain size of feldspar (mm) } & $\mathrm{NL}=-0.0494 \mathrm{x}+103.98$ & 0.0620 \\
\hline & $\mathrm{NL}=103.97 \mathrm{e}^{-0.0005 \mathrm{x}}$ & 0.0612 \\
\hline \multirow{2}{*}{ Mean grain size of quartz $(\mathrm{mm})$} & $\mathrm{NL}=-0.0463 \mathrm{x}+103.89$ & 0.0044 \\
\hline & $\mathrm{NL}=103.89 \mathrm{e}^{-0.0004 x}$ & 0.004 \\
\hline \multirow[t]{4}{*}{ Mean grain size of biotite (mm) } & $\mathrm{NL}=-0.484 \mathrm{x}+104.15$ & 0.0703 \\
\hline & $\mathrm{NL}=-0.4874 \operatorname{Ln}(\mathrm{x})+103.56$ & 0.1439 \\
\hline & $\mathrm{NL}=103.56 \mathrm{x}^{-0.0047}$ & 0.1418 \\
\hline & $\mathrm{NL}=104.15 \mathrm{e}^{-0.0046 \mathrm{x}}$ & 0.0691 \\
\hline \multirow[t]{4}{*}{ Mean grain size of rock (mm) } & $\mathrm{NL}=-0.0477 \mathrm{x}+103.93$ & 0.0115 \\
\hline & $\mathrm{NL}=-0.12 \operatorname{Ln}(\mathrm{x})+103.9$ & 0.0083 \\
\hline & $\mathrm{NL}=103.89 \mathrm{x}^{-0.0011}$ & 0.0080 \\
\hline & $\mathrm{NL}=103.92 \mathrm{e}^{-0.0005 \mathrm{x}}$ & 0.0111 \\
\hline \multirow[t]{4}{*}{ Max. grain size of plagioclase (mm) } & $\mathrm{NL}=-0.2046 \mathrm{x}+104.71$ & 0.1024 \\
\hline & $\mathrm{NL}=-0.8018 \operatorname{Ln}(\mathrm{x})+104.97$ & 0.088 \\
\hline & $\mathrm{NL}=104.98 \mathrm{x}^{-0.0077}$ & 0.0885 \\
\hline & $\mathrm{NL}=104.72 \mathrm{e}^{-0.002 \mathrm{x}}$ & 0.1028 \\
\hline \multirow[t]{2}{*}{ Max. grain size of feldspar (mm) } & $\mathrm{NL}=-0.0306 \mathrm{x}+104.05$ & 0.0728 \\
\hline & $\mathrm{NL}=104.05 \mathrm{e}^{-0.0003 \mathrm{x}}$ & 0.0714 \\
\hline \multirow[t]{2}{*}{ Max. grain size of quartz $(\mathrm{mm})$} & $\mathrm{NL}=-0.0684 \mathrm{x}+104.07$ & 0.0567 \\
\hline & $\mathrm{NL}=104.07 \mathrm{e}^{-0.0007 \mathrm{x}}$ & 0.0556 \\
\hline \multirow[t]{4}{*}{ Max. grain size of biotite (mm) } & $\mathrm{NL}=-0.0603 \mathrm{x}+103.95$ & 0.0067 \\
\hline & $\mathrm{NL}=-0.3684 \operatorname{Ln}(\mathrm{x})+104.05$ & 0.1129 \\
\hline & $\mathrm{NL}=104.04 \mathrm{x}^{-0.0035}$ & 0.1112 \\
\hline & $\mathrm{NL}=103.94 \mathrm{e}^{-0.0006 \mathrm{x}}$ & 0.0063 \\
\hline \multirow[t]{4}{*}{ Plagioclase content $(\%) \mathrm{x}$ its mean grain size $(\mathrm{mm})$} & $\mathrm{NL}=1.772 \mathrm{x}+102.94$ & 0.1312 \\
\hline & $\mathrm{NL}=0.5537 \operatorname{Ln}(\mathrm{x})+104.24$ & 0.0568 \\
\hline & $\mathrm{NL}=104.23 \mathrm{x}^{0.0053}$ & 0.0556 \\
\hline & $\mathrm{NL}=102.95 \mathrm{e}^{0.0169 \mathrm{x}}$ & 0.1294 \\
\hline
\end{tabular}


Table 7. continued.

\begin{tabular}{llr}
\hline Mineralogical properties & \multicolumn{1}{c}{ Regression equation } & $\mathrm{R}^{2}$ \\
\hline Feldspar content $(\%) \mathrm{x}$ its mean grain size $(\mathrm{mm})$ & $\mathrm{NL}=-0.1097 \mathrm{x}+103.96$ & 0.0547 \\
& $\mathrm{NL}=103.96 \mathrm{e}^{-0.001 \mathrm{x}}$ & 0.0539 \\
Quartz content $(\%) \mathrm{x}$ its mean grain size $(\mathrm{mm})$ & $\mathrm{NL}=-0.5339 \mathrm{x}+104.05$ & 0.0707 \\
& $\mathrm{NL}=104.04 \mathrm{e}^{-0.0051 \mathrm{x}}$ & 0.0697 \\
Biotite content $(\%) \mathrm{x}$ its mean grain size of $(\mathrm{mm})$ & $\mathrm{NL}=-5.156 \mathrm{x}+104.02$ & 0.0363 \\
& $\mathrm{NL}=-0.2878 \mathrm{Ln}(\mathrm{x})+102.79$ & 0.1328 \\
& $\mathrm{NL}=102.8 \mathrm{x}^{-0.0028}$ & 0.1315 \\
& $\mathrm{NL}=104.02 \mathrm{e}^{-0.049 \mathrm{x}}$ & 0.0354 \\
\hline
\end{tabular}

$\mathbf{N L}_{\mathbf{R 6}}(\mathbf{d B})=87.2765+0.2530 \mathrm{~A}+0.1270 \mathrm{~B}+1.0230 \mathrm{C}-0.0078 \mathrm{D}$

CR (\%): A: 35.95, B: 36.09, C: 16.91, D: 11.05 .

$\mathbf{N L}_{\mathbf{R} 7}(\mathbf{d B})=82.0047+0.2860 \mathrm{~A}+0.1546 \mathrm{~B}+0.7466 \mathrm{C}$

CR (\%) : A: 40.76, B : 44.07, C: 12.38 .

$\mathbf{N L}_{\mathbf{R 8}}(\mathbf{d B})=88.0695+0.2470 \mathrm{~A}+0.1157 \mathrm{~B}+1.3030 \mathrm{C}-0.0061 \mathrm{D}$

CR (\%) : A: 35.74, B : 33.48, C: 21.93, D: 8.85 .

$\mathbf{N L}_{\mathbf{R 9}}(\mathbf{d B})=86.9416+0.2738 \mathrm{~A}+0.1200 \mathrm{~B}+1.5088 \mathrm{C}-0.0115 \mathrm{D}$

CR (\%): A: 34.04, B: 29.84, C: 21.82, D: 14.30 .

In addition to developing the models relating to the operating variables, additional models were established for prediction of noise levels from rock properties. In the models, independent variables were denoted by the following; $\mathrm{X}_{1}$ : uniaxial strength $(\mathrm{MPa}), \mathrm{X}_{2}$ : density $\left(\mathrm{kN} / \mathrm{m}^{3}\right), \mathrm{X}_{3}$ : bending strength $(\mathrm{MPa}), \mathrm{X}_{4}$ : water absorption by volume $(\%), \mathrm{X}_{5}$ : porosity $(\%), \mathrm{X}_{6}$ : Schmidt hammer hardness, $\mathrm{X}_{7}$ : ultrasonic velocity $(\mathrm{m} / \mathrm{s}), \mathrm{X}_{8}$ : Shore hardness, $\mathrm{X}_{9}$ : Cerchar abrasion index (HV), $\mathrm{X}_{10}$ : microhardness, $\mathrm{X}_{11}$ : Mohs hardness, $\mathrm{X}_{12}$ : plagioclase content $(\%), \mathrm{X}_{13}$ : alkali feldspar content $(\%), \mathrm{X}_{14}$ : quartz content $(\%), \mathrm{X}_{15}$ : biotite content $(\%), \mathrm{X}_{16}$ : maximum grain size of plagioclase $(\mathrm{mm}), \mathrm{X}_{17}$ : maximum grain size of alkali feldspar $(\mathrm{mm}), \mathrm{X}_{18}$ : maximum grain size of quartz $(\mathrm{mm}), \mathrm{X}_{19}$ : maximum grain size of biotite $(\mathrm{mm}), \mathrm{X}_{20}$ : mean grain size of rock $(\mathrm{mm}), X_{21}$ : mean grain size of plagioclase $(\mathrm{mm}), \mathrm{X}_{22}$ : mean grain size of alkali feldspar $(\mathrm{mm}), \mathrm{X}_{23}$ : mean grain size of quartz $(\mathrm{mm}), \mathrm{X}_{24}$ : mean grain size of biotite $(\mathrm{mm})$. The determinant coefficients of the models are 1 and that means $100 \%$ of the variation of the experimental data are explained by the models built. The alternative models, where the physico-mechanical and mineralogical properties were considered together as independent variables are presented below (equations from 10 to 12 )

$$
\begin{gathered}
\text { NL }(\mathbf{d B})=97.5498+0.0129 \mathrm{X}_{1}-0.0591 \mathrm{X}_{8}-0.0013 \mathrm{X}_{10}+0.9859 \mathrm{X}_{11} \\
+0.0988 \mathrm{X}_{16}+1.7859 \mathrm{X}_{21}+0.0405 \mathrm{X}_{22}-0.5188 \mathrm{X}_{24} \\
\text { CR }(\%): \mathrm{X}_{1}: 24.78, \mathrm{X}_{8}: 13.93, \mathrm{X}_{10}: 1.39, \mathrm{X}_{11}: 19.20, \mathrm{X}_{16}: 4.66 \\
\mathrm{X}_{21}: 21.31, \mathrm{X}_{22} ; 6.16, \mathrm{X}_{24}: 8.57
\end{gathered}
$$




$$
\begin{gathered}
\text { NL }(\mathbf{d B})=89.9470+0.5331 \mathrm{X}_{2}+1.0033 \mathrm{X}_{4}-0.11713 \mathrm{X}_{15}-0.3340 \mathrm{X}_{16} \\
+0.8532 \mathrm{X}_{19}+0.3818 \mathrm{X}_{21}+0.0999 \mathrm{X}_{22}-2.2023 \mathrm{X}_{24} \cdot \\
\text { CR }(\%): \mathrm{X}_{2}: 17.36, \mathrm{X}_{4}: 5.31, \mathrm{X}_{15}: 8.67, \mathrm{X}_{16}: 10.13, \mathrm{X}_{19}: 22.42 \\
\mathrm{X}_{21}: 2.93, \mathrm{X}_{22}: 9.77, \mathrm{X}_{24}: 23.40 .
\end{gathered}
$$

$$
\begin{gathered}
\text { NL }(\mathbf{d B})=105.4205-0.01959 \mathrm{X}_{3}-1.5154 \mathrm{X}_{4}-1.0327 \mathrm{X}_{5}-0.0053 \mathrm{X}_{10} \\
\quad+0.0281 \mathrm{X}_{16}+1.9275 \mathrm{X}_{21}-0.1157 \mathrm{X}_{22}+0.2284 \mathrm{X}_{23} . \\
\text { CR }(\%): \mathrm{X}_{3}: 2.48, \mathrm{X}_{4}: 11.87, \mathrm{X}_{5}: 30.89, \mathrm{X}_{10}: 5.52, \mathrm{X}_{16}: 1.26 \\
\mathrm{X}_{21}: 21.86, \mathrm{X}_{22}: 16.72, \mathrm{X}_{23}: 9.40 .
\end{gathered}
$$

The models built only considering the physico-mechanical properties as independent variables are presented below (equations from 13 to 15). The determinant coefficients of these models are also 1 confirming that $100 \%$ of the variation of the experimental data is explained by the models established. It was observed that among the physico-mechanical properties, bending strength, porosity, Schmidt hardness, Mohs hardness and microhardness involved in the best models developed.

$$
\begin{gathered}
\text { NL }(\mathbf{d B})=100.0677+0.0136 \mathrm{X}_{1}-0.0167 \mathrm{X}_{3}-4.5915 \mathrm{X}_{4}+0.0189 \mathrm{X}_{5} \\
\quad+0.0341 \mathrm{X}_{6}-0.0018 \mathrm{X}_{7}+0.0132 \mathrm{X}_{10}-0.9530 \mathrm{X}_{11} . \\
\text { CR }(\%): \mathrm{X}_{1}: 24.22, \mathrm{X}_{3}: 2.07, \mathrm{X}_{4}: 35.04, \mathrm{X}_{5}: 0.55, \mathrm{X}_{6}: 7.20 \\
\mathrm{X}_{7}: 0.38, \mathrm{X}_{10} ; 13.34, \mathrm{X}_{11}: 17.20 .
\end{gathered}
$$

$$
\begin{gathered}
\text { NL }(\mathbf{d B})=138.5507-0.2550 \mathrm{X}_{3}-1.7978 \mathrm{X}_{4}-0.2650 \mathrm{X}_{5}+0.0321 \mathrm{X}_{6} \\
\quad-0.0946 \mathrm{X}_{8}-3.3379 \mathrm{X}_{9}+0.0147 \mathrm{X}_{10}-2.9525 \mathrm{X}_{11} . \\
\text { CR }(\%): \mathrm{X}_{3}: 15.43, \mathrm{X}_{4}: 6.72, \mathrm{X}_{5}: 3.78, \mathrm{X}_{6}: 3.31, \mathrm{X}_{8}: 10.12 \\
\mathrm{X}_{9}: 27.24, \mathrm{X}_{10} ; 7.27, \mathrm{X}_{11}: 26.09 .
\end{gathered}
$$

$$
\begin{aligned}
\mathbf{N L}(\mathbf{d B})= & 102.6679+0.0140 \mathrm{X}_{1}-0.3018 \mathrm{X}_{3}-1.4934 \mathrm{X}_{5}-0.3228 \mathrm{X}_{6} 0.0033 \mathrm{X}_{7} \\
& -1.4044 \mathrm{X}_{9}+0.0494 \mathrm{X}_{10}-2.0235 \mathrm{X}_{11} .
\end{aligned}
$$

CR $(\%): \mathrm{X}_{1}: 6.90, \mathrm{X}_{3}: 10.28, \mathrm{X}_{5}: 12.00, \mathrm{X}_{6}: 18.76, \mathrm{X}_{7}: 31.84$,

$$
\mathrm{X}_{9}: 6.45, \mathrm{X}_{10} ; 13.77, \mathrm{X}_{11}: 10.07 \text {. }
$$

The models derived from only mineralogical properties of the rock are presented below (equations from 16 to 18). The determinant coefficients of these models are 1 . This value implies also that $100 \%$ of the variation of the experimental data is explained by the models derived. It was seen that among the mineralogical properties, quartz and biotite involved in the best models derived.

$$
\begin{aligned}
\mathbf{N L}(\mathbf{d B})= & 102.4950-0.0976 \mathrm{X}_{14}-0.0373 \mathrm{X}_{15}+0.0349 \mathrm{X}_{16}+0.0930 \mathrm{X}_{17} \\
& +1.8271 \mathrm{X}_{21}-0.0252 \mathrm{X}_{22}+0.4451 \mathrm{X}_{23}-2.1955 \mathrm{X}_{24} .
\end{aligned}
$$

CR $(\%): \mathrm{X}_{14}: 26.75, \mathrm{X}_{15}: 2.81, \mathrm{X}_{16}: 1.08, \mathrm{X}_{17}: 16.18, \mathrm{X}_{21}: 14.28$,

$$
\mathrm{X}_{22}: 2.51, \mathrm{X}_{23} ; 12.62, \mathrm{X}_{24}: 23.76 \text {. }
$$




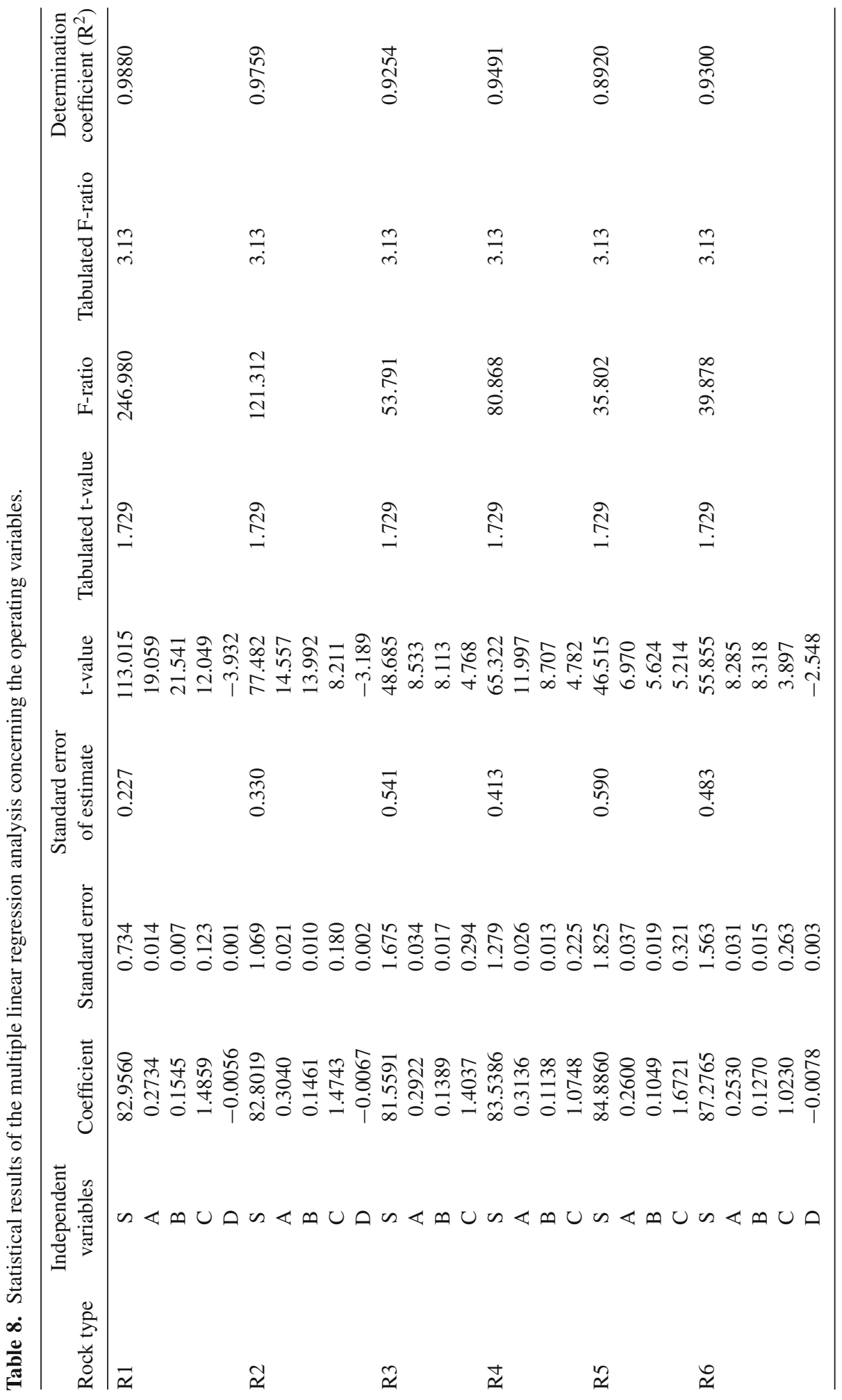




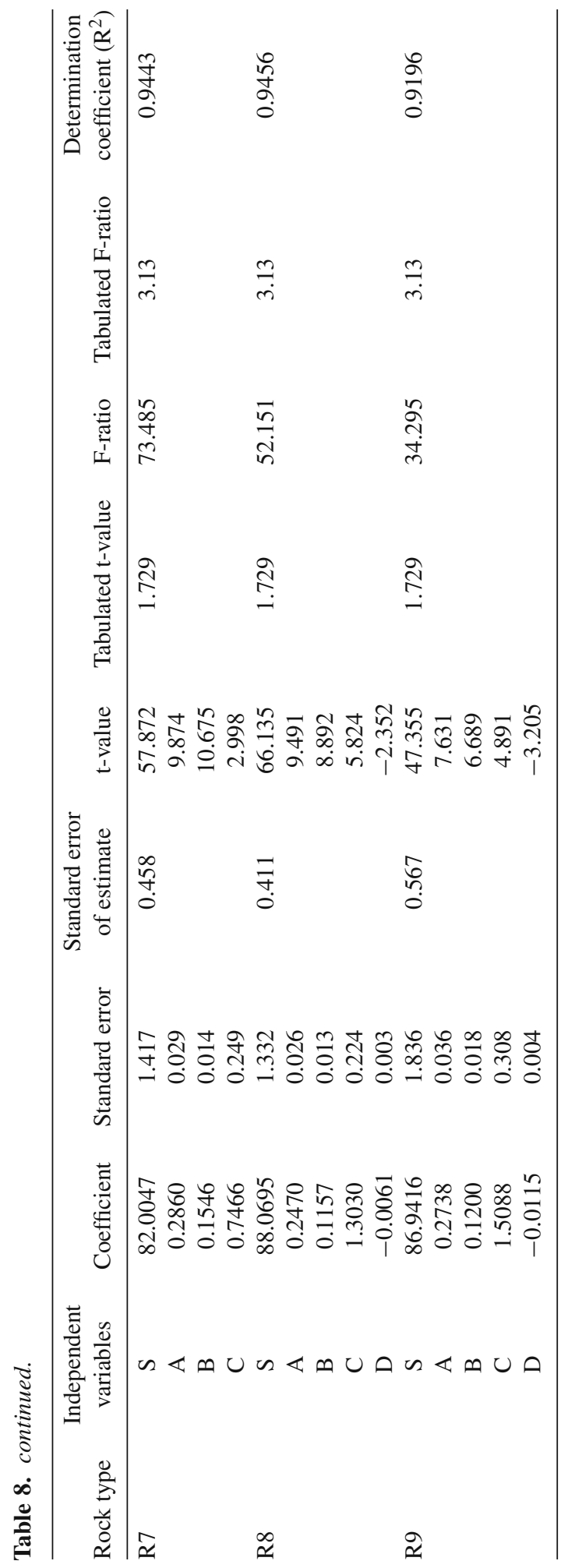




$$
\begin{gathered}
\text { NL }(\mathbf{d B})=111.0637-0.1032 \mathrm{X}_{12}-0.0457 \mathrm{X}_{13}-0.0652 \mathrm{X}_{14}-0.0723 \mathrm{X}_{15} \\
\quad-0.1926 \mathrm{X}_{16}+0.0123 \mathrm{X}_{17}-0.0430 \mathrm{X}_{18}-0.1419 \mathrm{X}_{19} . \\
\text { CR }(\%): \mathrm{X}_{12}: 34.06, \mathrm{X}_{13}: 22.87, \mathrm{X}_{14} ; 20.16, \mathrm{X}_{15}: 6.15, \mathrm{X}_{16}: 6.71, \\
\mathrm{X}_{17}: 2.42, \mathrm{X}_{18}: 3.34, \mathrm{X}_{19}: 4.29 .
\end{gathered}
$$

$$
\begin{aligned}
\mathbf{N L}(\mathbf{d B})= & 105.5257+0.0102 \mathrm{X}_{12}+0.1264 \mathrm{X}_{13}-0.2471 \mathrm{X}_{14}-0.1155 \mathrm{X}_{15} \\
& +0.3398 \mathrm{X}_{21}+0.0442 \mathrm{X}_{22}+1.1789 \mathrm{X}_{23}-5.0106 \mathrm{X}_{24} .
\end{aligned}
$$

CR $(\%): \mathrm{X}_{12}: 1.30, \mathrm{X}_{13}: 24.37, \mathrm{X}_{14} ; 29.41, \mathrm{X}_{15}: 3.78, \mathrm{X}_{21}: 1.15$,

$$
\mathrm{X}_{22}: 1.91, \mathrm{X}_{23}: 14.52, \mathrm{X}_{24}: 23.55 \text {. }
$$

Although the models (from 10 to 18 ) have the highest determination coefficients, the number of the independent variables involved in the models may lead to difficulties for the practical applications. In order to facilitate the applicability of the model, therefore, an alternative model for prediction of the noise level was developed. For practical considerations, the following equation (19) was selected among the all possible equations. As seen, uniaxial compressive strength and water absorption were included in the model.

$$
\begin{aligned}
& \text { NL }(\mathbf{d B})=102.0759+0.0137 \mathrm{X}_{1}-2.0036 \mathrm{X}_{4} . \\
& \text { CR }(\%): \mathrm{X}_{1}: 61.52, \mathrm{X}_{4}: 38.48 .
\end{aligned}
$$

\subsection{Verification of the models}

The statistical results of the nine models (equations from 1 to 9) are given in table 8 . The models built from material properties (excluding the model (19)) were not tested since the determination coefficients of these models are 1 . That means $100 \%$ of the variation of the experimental data
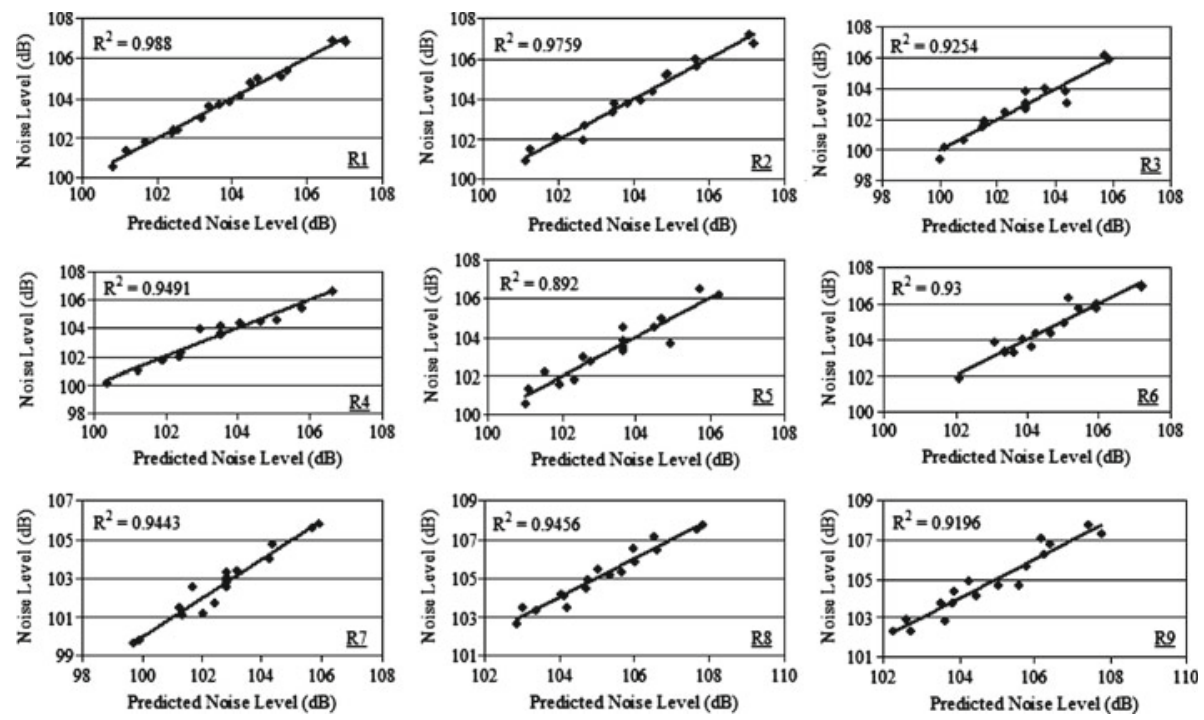

Figure 8. Predicted noise level vs. observed noise level. 

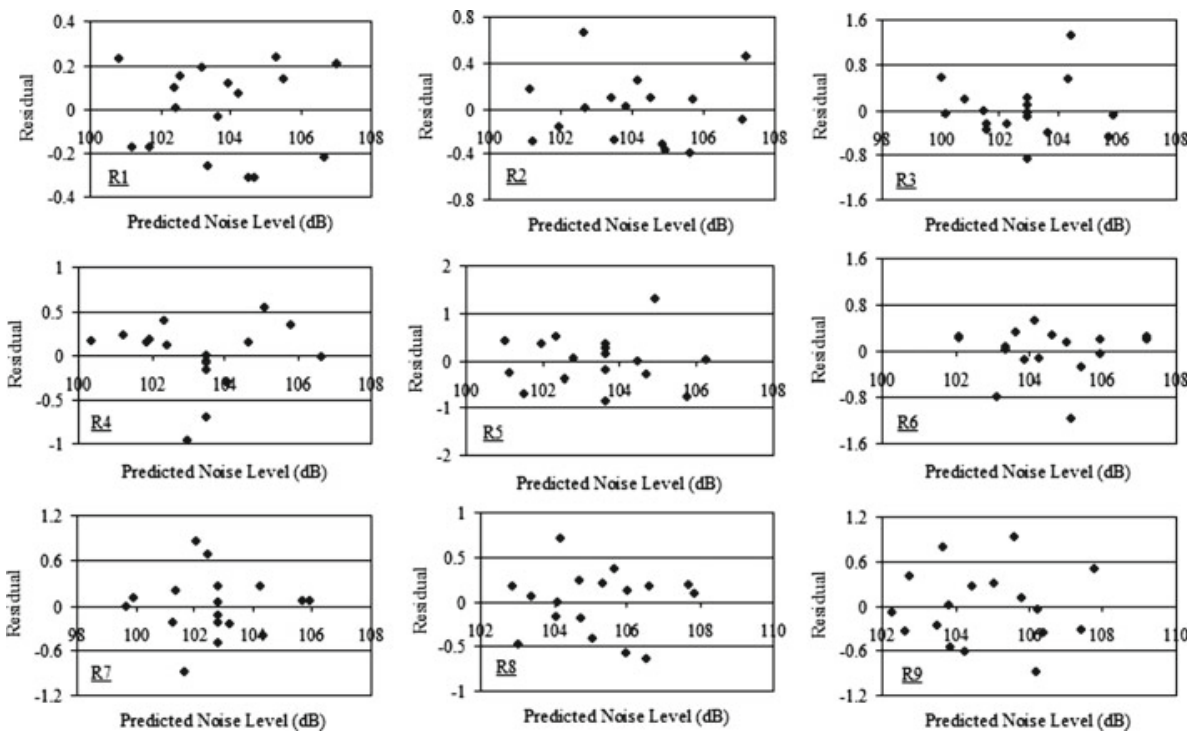

Figure 9. Residuals against the predicted noise level.

is explained by the equations. In verification tests, a $95 \%$ level of confidence was chosen. The prediction capability and/or verification of the derived models was tested by the scatter diagrams of the observed and predicted values, the status of determination coefficients (see also figure 8) and the F-test, while each operating variable involved in the models was verified by the t-test. If the calculated F-and t-values are greater than those of tabulated, it confirms that the related models and each operating variable involved in the models are statistically significant. In other words, it can be concluded that the derived models are valid. From table 8 and figure 8 , it was seen that the $\mathrm{R}^{2}$ of the models are greater than 0.90 except for $\mathrm{R} 7$. The $\mathrm{R}^{2}$ of the model for $\mathrm{R} 7$ is 0.89 . These values indicate that there is a high degree of relationship between the predicted and observed noise levels. As can also be seen in table 8, all calculated F-and t-values are greater than those of tabulated values suggesting that the derived models and each operating variables involved in the models are statistically valid. Additionally, residual analysis was used to check the assumptions of the models. The residuals are the difference between the real and the predicted data. The graphs of the residuals showed that there is nearly symmetrical distribution on the line
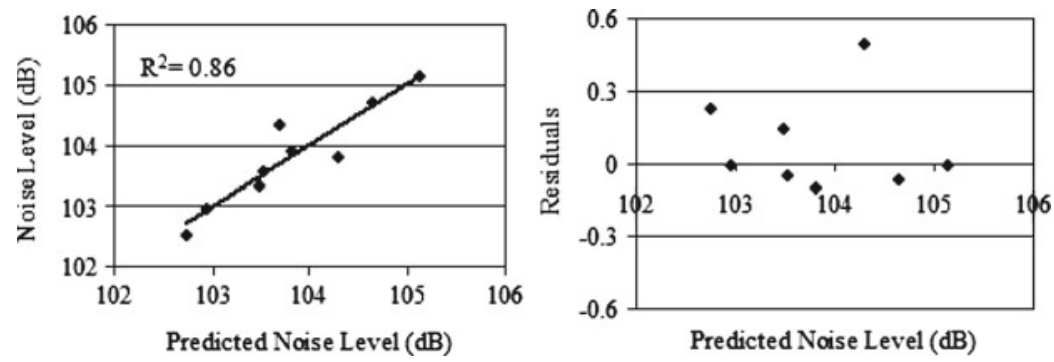

Figure 10. Predicted noise level from material properties vs. observed noise level, and residuals against the predicted noise level from material properties. 
Table 9. Statistical results of the multiple linear regression analysis concerning material properties.

\begin{tabular}{lccc}
\hline & $\mathrm{S}$ & $\mathrm{X}_{1}$ & $\mathrm{X}_{4}$ \\
\hline Coefficient & 102.0759 & 0.01373 & -2.0036 \\
Standard error & 0.4286 & 0.0025 & 0.5755 \\
Standard error of estimate & & 0.3597 & -3.4817 \\
t-value & 238.1763 & 5.5662 & \\
Tabulated t-value & & 1.86 & \\
F-ratio & & 18.2203 & \\
Tabulated F-ratio & & 5.32 & \\
Determination coefficient $\left(\mathrm{R}^{2}\right)$ & & 0.8586 & \\
\hline
\end{tabular}

verifying that the models built are statistically valid (figure 9). Furthermore, the determination coefficient of the simple and practical equation (19) implies that $86 \%$ of the variation in the data is explained by the equation (19) (figure 10). As can be seen from table 9, the model is statistically valid in terms of $\mathrm{t}$-and $\mathrm{F}$ - tests as well. The residual plot also confirms the accuracy of the model developed (see figure 10).

\section{Conclusions}

An experimental and statistical study on noise level generated during the sawing of granites using circular diamond sawblades was presented in this study. It was observed that increasing of peripheral speed, traverse speed and cutting depth resulted in an increase in noise levels recorded for all rocks. Unlike the behaviour of other operating variables, a decreasing trend for noise levels was initially observed with the increasing of flow rate of cooling fluid. A relation between uniaxial compressive strength, density and noise levels was determined for all rocks. Modelling results also revealed that the noise level can be effectively predicted from both the operating variables and rock properties. The verification methods confirmed the correctness of the developed models that offer promising potential for future applications.

\section{Acknowledgements}

The authors would like to thank the Scientific Research Fund of Karadeniz Technical University for the financial support (No. 2009.112.008.3). The authors are also thankful to Granitaş A.Ş. stone processing company for supporting this research by providing dimensioned rock samples for the sawing experiments.

\section{References}

Ataei M, Mikaeil R, Hoseinie H S and Hosseini M S 2012 Fuzzy analytical hierarchy process approach for ranking the sawability of carbonate rock. Int. J. Rock Mech. Min. 50: 83-93

Atici U and Ersoy A 2009 Correlation of specific energy of cutting saws and drilling bits with rock brittleness and destruction energy. J. Mater. Process. Technol. 209: 2602-2612

Buyuksagis S I 2007 Effect of cutting mode on the sawability of granites using segmented circular diamond sawblade. J. Mater. Process. Technol. 183: 399-406

Ersoy A and Atic1 U 2004 Performance characteristics of circular diamond saws in cutting different types of rocks. Diam. Relat. Mater. 13: 22-37 
Fener M, Kahraman S and Ozder O M 2007 Performance prediction of circular diamond saws from mechanical rock properties in cutting carbonate rocks. Rock Mech. Rock Eng. 40(5): 505-517

ISRM 1981 Rock characterization testing and monitoring, suggested methods, in: E T Brown (ed.), Pergamon Press, Oxford, UK, 211 p.

Kahraman S, Fener M and Gunaydin O 2004 Predicting the sawability of carbonate rocks using multiple curvilinear regression analysis. Int. J. Rock Mech. Min. 41(7): 123-1131

Muro T and Tran T D 2004 Regression analysis of the characteristics of vibro-cutting blade for tuffaceous rock. J. Terramechanics 40: 191-219

Rojek J, Onate E, Labra C and Kargl H 2011 Discrete element simulation of rock cutting. Int. J. Rock Mech. Min. 48: 996-1010

Y1lmaz G N, Goktan M R and Kibici Y 2011 An investigation of the petrographic and physico-mechanical properties of true granites influencing diamond tool wear performance, and development of a new wear index. Wear 271: 960-969

Yurdakul M and Akdas H 2012 Prediction of specific cutting energy for large diameter circular saws during natural stone cutting. Int. J. Rock Mech. Min. 53: 38-44 